\title{
Hyponatremia is a potential predictor of progression in radiation-induced brain necrosis: a retrospective study
}

\author{
Huan Liao ${ }^{1 \dagger}$, Zhuoting Zhu ${ }^{2 \dagger}$, Xiaoming Rong ${ }^{1}$, Hongxuan Wang ${ }^{1}$ and Ying Peng ${ }^{1,3^{*}}$ (D)
}

\begin{abstract}
Background: To investigate the prognostic value of hyponatremia, defined as serum sodium level $<135 \mathrm{mEq} / \mathrm{L}$, in radiation-induced brain necrosis (RN) patients.

Methods: We performed a retrospective analysis of the RN patients (The patients included in our study had a history of primary cancers including nasopharyngeal carcinoma/glioma/oral cancer and received radiotherapy previously and then were diagnosed with RN) treated in Sun yat-sen Memorial Hospital from January 2013 to August 2015. Patients without cranial magnetic resonance imaging (MRI) scan and serum sodium data were excluded. Progression was identified when the increase of edema area $\geq 25 \%$ on the MRI taken in six months comparing with those taken at the baseline. Factors that might associate with prognosis of RN were collected. Multivariable logistic regression analyses were used to identify potential predictors.
\end{abstract}

Results: We total included 135 patients, 32 (23.7\%) of them with hyponatremia and 36 (26.7\%) with RN progression. Percentage of progression was roughly three fold in hyponatremia patients compared with nonhyponatremia patients (53.1\% versus $18.4 \%)$, translating into a 5 -fold increased odds ratio $(P<0.001)$. Multivariable analyses identified hyponatremia as a potential predictor of progression (OR, 4.82; 95\% Cl [1.94-11.94]; $P=0.001)$.

Conclusions: Hyponatremia was identified as a potential predictor for the progression of patients with RN. Hyponatremia management in patients with $\mathrm{RN}$ should be paid much more concern in clinical practice.

Keywords: Hyponatremia, Radiation-induced brain necrosis

\section{Background}

Radiotherapy is the standard treatment for nasopharyngeal carcinoma (NPC) and various brain tumors. NPC is the third most common malignant tumor in men in certain regions of East Asia, with an incidence of $15 / 100,000$ to $50 / 100000$ [1]. Moreover, since radiotherapy is invariably associated with radiation exposure of surrounding healthy tissues [2], nearly 100,000 primary and metastatic brain neoplasm patients/year survive long enough ( $>$ half a year) to suffer radiation-induced brain necrosis

\footnotetext{
*Correspondence: docpengy123@163.com

${ }^{+}$Huan Liao and Zhuoting Zhu contributed equally to this work.

'Department of Neurology, Sun Yat-sen Memorial Hospital, Sun Yat-Sen University, No. 107 West Yanjiang Road, Guangzhou 510120, China

${ }_{3}^{3}$ Guangdong Provincial Key Laboratory of Malignant Tumor Epigenetics and Gene Regulation, Sun Yat-sen Memorial Hospital, Sun Yat-sen University, Guangzhou, China

Full list of author information is available at the end of the article
}

(RN) in the US [3]. RN is a progressive disease, rendering impairments in attention, memory and executive function, which results in decreased patient quality of life [3].

There is consistent evidence demonstrating that hyponatremia not only acts as risk factors in various diseases such as cancer [4] and heart failure [5], but also has close relationship with central nervous system (CNS) diseases, such as stroke [6], subarachnoid hemorrhage $[7,8]$ and meningitis $[9,10]$, through inducing longer hospital stay, increased mortality, and raised complications [11-14]. In neurointensive care, it is usually the development of delayed cerebral infarctions, seizures, and cerebral edema that connects hyponatremia to prognosis [6]. Documentaries also suggest that hyponatremia probably serves as an onlooker reflecting the severity of diseases since it might be preexisting $[6,15,16]$, which might be induced by cerebral salt wasting syndrome(CWS) and the syndrome

(C) The Author(s). 2018 Open Access This article is distributed under the terms of the Creative Commons Attribution 4.0 International License (http://creativecommons.org/licenses/by/4.0/), which permits unrestricted use, distribution, and 
of inappropriate antidiuretic hormone secretion(SIADH) $[13,17,18]$.

Whereas, for RN, whether hyponatremia is related to the prognosis of $\mathrm{RN}$ has not been addressed. In this study, by facilitating a comprehensive coverage of patient characteristics, we aimed to clarify factors associated with prognosis of RN.

\section{Methods}

\section{Patient selection}

The study was approved by the Institutional Review Board of Sun yat-sen Memorial Hospital. We retrospectively collected the data of patients diagnosed with RN (The patients included in our study had a history of primary cancers including nasopharyngeal carcinoma/glioma/oral cancer and received radiotherapy previously and then were diagnosed with RN) and treated at Department of Neurology, Sun Yat-Sen Memorial Hospital from January, 2013 to August, 2015. The eligible criteria were listed as following: (1) Patients diagnosed with RN between January, 2013 and August, 2015 in the Department of Neurology, Sun Yat-Sen Memorial Hospital; (2) Patients with cranial MRI scan at baseline (the date of the first MRI performed for the diagnosis of RN for the patients in Sun Yat-Sen Memorial Hospital between January, 2013 and August, 2015) and six-month follow-up; (3) Available data of serum sodium during the period between the time of baseline and six-month follow-up.

\section{Clinical details}

Patients' characteristics including age, sex, in-hospital days, history of hypertension, epilepsy, dyslipidemia, cerebral infarction, pulmonary infection, paralysis of cranial nerves, nasopharyngeal carcinoma, glioma, oral cancer and baseline result of cranial MRI were retrieved from our institutional prospective electronic medical records. Serum sodium values were measured by use of Ion Selective Electrodes with automated specimen dilution (Cobas Integra 800, Roche) and the lowest values within the time period mentioned above were selected for analysis. Hyponatremia was defined as serum sodium values less than $135 \mathrm{mmol} / \mathrm{L}$ according to Hyponatremia Guidelines in Europe in 2014 [19] and was corrected by using hypertonic saline infusions $(\mathrm{NaCl}, 3 \%)$ or $0.9 \%$ sodium infusions aiming at an increase of $\leq 8 \mathrm{mg} / \mathrm{dL}$ per $24 \mathrm{~h}$. In addition, all patients were treated with a conventional corticosteroid regimen as described in our prior study [20].

\section{MRI acquisition and analysis}

Diagnosis of RN was made by clinical history and MRI performance (Sonata; $1.5 \mathrm{~T}$; Siemens), which included T1-weighted gadolinium contrast-enhanced and T2-weighted image. The MRI recognition of $\mathrm{RN}$ was referred to a prior study [21]. Typically, the most common feature of MRI in patients with RN is that the appearance of focal necrosis and finger like edema displays low signal intensity on T1WI while high signal on T2WI. We draw the edge of the maximum area of each edema manually which was then calculated automatically by software Volume Viewer 2(GE, AW Suite 2.0, 6.5.1.z). Two neuroradiologists who were blinded to clinical data reviewed the scans independently. Particularly, edema area was counted as the mean value of two measurements. Moreover, in cases of discrepancies, a second consensus analysis was made by the third author. Follow-up MRIs at six months were used to evaluate the end-point. The end-point is the edema progression occurrence, based on our prior methods [20]. Briefly, the edema progression occurrence was identified when the increase of edema area $>25 \%$.

\section{Statistical analysis}

Student's $t$ test (presented as mean \pm SD) or Mann-Whitney test (median, range) was used to compare continuous variables, while Pearson Chi-square or Fisher's exact test was used for the comparison of categorical data where appropriate. Multivariable logistic analyses were calculated to investigate independent risk factors for progression. Parameters reaching a statistical trend in univariable analysis $(P<0.30)$ were included into the multivariable models. Collinearity was examined using variance inflation factors (VIF) procedure. Statistical significance was defined as a $P$ value $<0.05$ at 2 -sided. All statistical analyses were performed with SPSS version 22.0 (SPSS Inc).

\section{Results}

A total of 135 patients were included in our study (Fig. 1), with a mean age of $50.9 \pm 10.0$ years old. 103 patients $(76.3 \%)$ were male and 32 (23.7\%) were female. Among the patients, 126 (93.3\%) had a history of nasopharyngeal carcinoma, 8 (6.0\%) had glioma and $1(0.7 \%)$ had oral cancer. The median timing of the lowest concentration was one month after the first MRI performed. The mean of the lowest serum sodium values within the six-month period of each patients were $136.7 \pm 5.1 \mathrm{mmol} / \mathrm{L}$. Out of 135 patients enrolled for our analysis, 32 patients (23.7\%) had hyponatremia and 36 (26.7\%) occurred edema progression (increase of edema area $>25 \%$ ) within a six-month follow-up.

Table 1 shows the comparison of clinical characteristics between patients with and without hyponatremia. Hyponatremia patients were showing statistical trends to have more male gender $(87.5 \%$ versus $72.8 \%, P=0.088)$ and more frequent existing of pulmonary infection (12.5\% versus $3.0 \%, P=0.093)$. In other aspects of clinical characteristics, there were no significant differences between these two groups. 


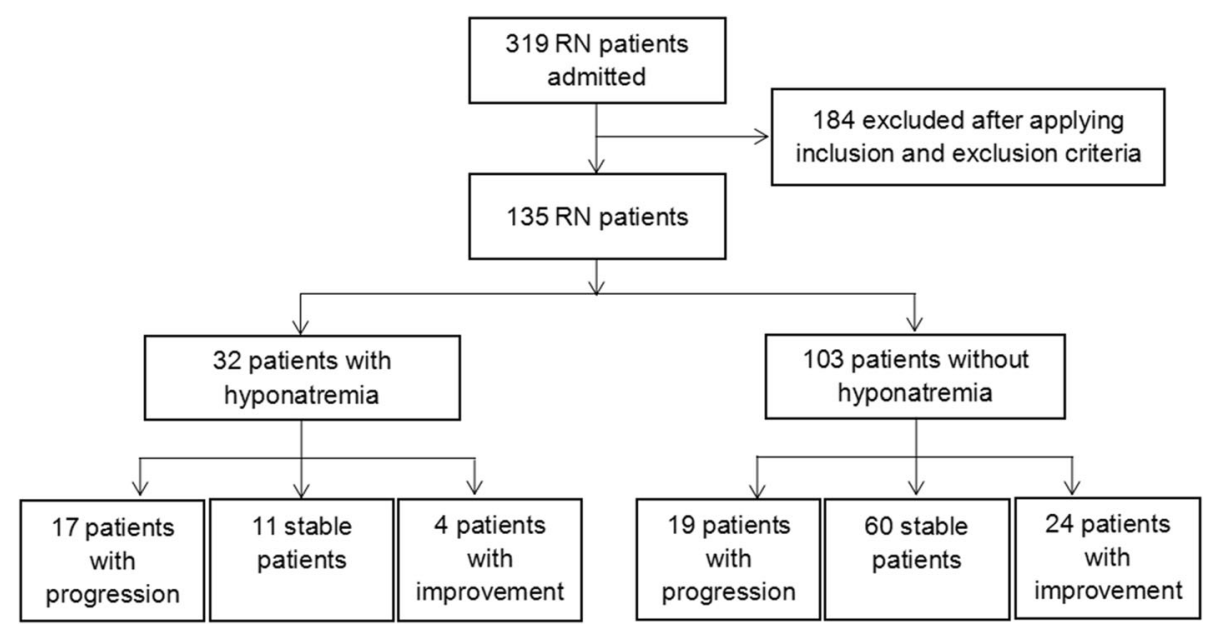

Fig. 1 Study flow chart

Table 2 displays the clinical characteristics of the patients divided into 2 groups according to the presence of edema progression. Patients whose cranial MRI indicating progression were more likely to have lower serum sodium concentration $(132.9 \pm 6.2 \mathrm{mmol} / \mathrm{L}$ versus $138.1 \pm 3.8 \mathrm{mmol} / \mathrm{L}$, $P<0.001)$ and higher incidence of medical history on hypertension $(27.8 \%$ versus $13.1 \%, P=0.045)$.

Percentage of progression was roughly three fold in hyponatremia compared with nonhyponatremia patients $(53.1 \% ; n=17$ versus $18.4 \% ; n=19)$, translating into a 5 -fold increased odds ratio (95\% CI, [2.13, 11.77]; $P<0.001)$. Multivariable analyses identified hyponatremia as an independent risk factor of progression (OR, 4.82; 95\% CI, [1.94, 11.94]; $P=0.001$ ), after adjustment with covariables including age, in-hospital days, history of hypertension, cerebral infarction, pulmonary infection and edema area in baseline MRI scan, which were found no collinearity existing (Table 3).

\section{Discussion}

To the best of our knowledge, this is the first retrospective study to document the association between hyponatremia and outcome of cranial MRI in RN patients. Our findings show that hyponatremia is a potential predictor of progression of RN.

It's very common to have hyponatremia among inpatients and outpatients, with prevalence ranging from 11 to $28.2 \%$ [22]. In our study, we found $23.7 \%$ of the enrolled

Table 1 Comparison of characteristics between patients with or without hyponatremia

\begin{tabular}{llll}
\hline Characteristics & $\begin{array}{l}\text { Hyponatremia } \\
n=32\end{array}$ & $\begin{array}{l}\text { Nonhyponatremia } \\
n=103\end{array}$ \\
\hline Serum sodium, mmol/L (mean \pm SD) & $129.3 \pm 4.3$ & $139.0 \pm 2.4$ & $<0.001$ \\
Age, yrs. (mean \pm SD) & $52.0 \pm 9.8$ & $50.5 \pm 10.0$ & $75(72.8 \%)$ \\
Male & $28(87.5 \%)$ & $7.5(1.8-23)$ & 0.465 \\
In-hospital days (M) & $8.3(4-20)$ & $16(15.5 \%)$ & 0.548 \\
Hypertension & $7(21.9 \%)$ & $15(14.6 \%)$ & 0.405 \\
Epilepsy & $3(9.4 \%)$ & $11(9.7 \%)$ & 0.648 \\
Dyslipidemia & $4(12.5 \%)$ & $8(7.8 \%)$ & 1.000 \\
Cerebral infarction & $5(15.6 \%)$ & $3(3.0 \%)$ & $16(15.5 \%)$ \\
Pulmonary infection & $4(12.5 \%)$ & $95(92.2 \%)$ & 0.330 \\
Paralysis of cranial nerves & $7(21.9 \%)$ & $7(6.8 \%)$ & 0.093 \\
Nasopharyngeal carcinoma & $31(96.9 \%)$ & $1(1.0 \%)$ \\
Glioma & $1(3.1 \%)$ & $5.3 \pm 3.8$ \\
Oral cancer & $0(0 \%)$ & $10.4 \pm 6.6$ \\
Baseline T1-weight gadolinium-contrast area (cm2)(mean \pm SD) & $5.1 \pm 2.3$ & 0.607 \\
BaselineT2-weighted area (cm2) (mean \pm SD) & $10.6 \pm 5.5$ & 0.734 \\
\hline
\end{tabular}


Table 2 Comparison of characteristics between patients with or without progression

\begin{tabular}{llll}
\hline Characteristics & $\begin{array}{l}\text { With progression } \\
n=36\end{array}$ & $\begin{array}{l}\text { Without progression } \\
n=99\end{array}$ \\
\hline Serum sodium, mmol/L (mean \pm SD) & $132.9 \pm 6.2$ & $138.1 \pm 3.8$ & $<0.001$ \\
Age, yrs. (mean \pm SD) & $52.7 \pm 11.0$ & $50.2 \pm 9.5$ & $76(76.8 \%)$ \\
Male & $27(75 \%)$ & $7.3(1.8-22)$ & 0.207 \\
In-hospital days (M) & $8.3(4.5-23)$ & $13(13.1 \%)$ & 0.831 \\
Hypertension & $10(27.8 \%)$ & $14(14.1 \%)$ & 119 \\
Epilepsy & $4(11.1 \%)$ & $12(12.1 \%)$ \\
Dyslipidemia & $3(8.3 \%)$ & $7(7.1 \%)$ \\
Cerebral infarction & $6(16.7 \%)$ & $3(3.0 \%)$ & 0.045 \\
Pulmonary infection & $4(11.1 \%)$ & $16(16.2 \%)$ \\
Paralysis of cranial nerves & $7(19.4 \%)$ & $93(94.0 \%)$ \\
Nasopharyngeal carcinoma & $33(91.7 \%)$ & $5(5.0 \%)$ \\
Glioma & $3(8.3 \%)$ & $1(1.0 \%)$ & 0.864 \\
Oral cancer & $0(0 \%)$ & $5.5 \pm 3.8$ \\
Baseline T1-weight gadolinium-contrast area (cm2)(mean \pm SD) & $4.5 \pm 2.4$ & $10.8 \pm 6.4$ \\
BaselineT2-weighted area (cm2) (mean \pm SD) & $9.3 \pm 5.9$ & 0.152 \\
\hline
\end{tabular}

$M$ median, $S D$ standard deviation

RN patients with hyponatremia. Hyponatremia has acquired broad recognition as a predictor being associated with poor outcome in various diseases [23, 24]. Two main hypothetical mechanisms of hyponatremia impacting on prognosis in disorders of CNS were SIADH and CSW [25]. Excessive antidiuretic hormone (ADH) release causing renal water reabsorption and expansion of the extracellular fluid volume (ECF) was the primary pathogenic mechanism underlying SIADH. When hypothalamus and pituitarium were under pressure or got injured, $\mathrm{ADH}$ would be secreted abnormally, causing hyponatremia. Meantime, the mechanism by which RN leads to renal salt wasting is also poorly understood. The most probable process involved central elaboration of a circulating natriuretic factor and/or disruption of neural input into the kidney. Atrial natriuretic peptide (ANP), brain natriuretic peptide (BNP), c-type natriuretic peptide (CNP) and ouabainlike compound (OLC) had been demonstrated to play a vital role in CSW [26-30], whose releasing also was related to the disruption of hypothalamus and pituitarium. Of note, it is important to make an accurate diagnosis because the treatment of each condition is quite different. Fluid restriction is the treatment of choice in patients with SIADH while vigorous salt replacement is required in patients with CSW. Existing data suggested that fluid restriction was very likely to worsen the underlying neurological condition in the setting of CSW, even cause cerebral infarction [31], acute symptomatic seizures [32] or death. Moreover, the clinical symptoms of vomiting and headache, were often regarded as the results of intracranial hypertension caused by brain edema in $\mathrm{RN}$ patients, thus mannitol was usually applied to alleviate the symptom. Nevertheless, in fact, the symptoms could not get remission sometimes, which implied that

Table 3 The logistic regression for the relationship between progression of RN and factors

\begin{tabular}{|c|c|c|c|c|c|}
\hline & \multirow[t]{2}{*}{ Progression } & \multicolumn{2}{|l|}{ Unadjusted } & \multicolumn{2}{|l|}{ Adjusted } \\
\hline & & OR $(95 \% \mathrm{Cl})$ & $P$-Value & OR $(95 \% \mathrm{Cl})$ & $P$-Value \\
\hline Hyponatremia $(n=32)$ & $17(53.1 \%)$ & $5.01(2.13 ; 11.77)$ & $<0.001$ & $4.82(1.94 ; 11.94)$ & 0.001 \\
\hline Age & - & - & & $1.01(0.97 ; 1.06)$ & 0.657 \\
\hline In-hospital days & - & - & & $1.05(0.95 ; 1.16)$ & 0.350 \\
\hline Hypertension ( $n=23)$ & $10(43.5 \%)$ & - & & $2.17(0.71 ; 6.60)$ & 0.172 \\
\hline Cerebral infarction $(n=13)$ & $6(46.2 \%)$ & - & & $2.04(0.47 ; 8.82)$ & 0.342 \\
\hline Pulmonary infection $(n=7)$ & $4(57.1 \%)$ & - & & $3.46(0.53 ; 22.43)$ & 0.194 \\
\hline T1-weight gadolinium-contrast area & - & - & & $1.10(0.70 ; 1.73)$ & 0.672 \\
\hline T2-weighted area & - & - & & $0.88(0.69 ; 1.12)$ & 0.299 \\
\hline
\end{tabular}

$O R$ odds ratio, $\mathrm{Cl}$ confidence interval 
the symptoms were not caused by brain edema, but very likely by hyponatremia [33]. The information above concludes that, on one hand, when doing irradiation therapy for the primary tumors, avoiding radiation exposure of hypothalamus and pituitarium if possible may effectively reduce the occurrence of hyponatremia from the source $[34,35]$. On the other hand, more proper dehydrants instead of those dehydrants which can cause hyponatremia should be chosen when dealing with brain edema of RN. Better management of hyponatremia in RN patients needs more attention.

There were several studies indicating that inflammatory cytokines such as Interleukin 6 (IL-6) [36] and Interleukin 1 beta (IL-1 $\beta$ ) [37] play vital roles in the nonosmotic release of $\mathrm{ADH}$. Under inflammatory conditions, this mechanism may be responsible for the occurrence of hyponatremia [38]. Interestingly, the mechanism of RN involves damage to the immune system [39]. In addition, previous studies found that after radiation, microglia in mouse brain tissue could secrete several inflammatory cytokines including IL-6, IL-1 $\beta$, tumor necrosis factor alpha (TNF- $\alpha$ ) and cyclooxygenase-2 (COX-2) [40]. Therefore, we speculate disorder of immune system could render the development of hyponatremia in patients with $\mathrm{RN}$, which should be demonstrated by further studies.

However, despite these postulated pathophysiological considerations of hyponatremia-induced impacts on prognosis, it remains unsolved if hyponatremia merely stands for an onlooker of diseases, that is, preexisting polymedication or comorbidity mirroring worse overall status, which itself maybe responsible for poor prognosis. For instance, hyponatremia was independently associated with anemia on hospital admission, mentioning that hyponatremia was probably preexisting condition rather than developing acutely [6]. Accordingly, in our study, hypertension was also found to be associated with progression of $\mathrm{RN}$. This need to be paid more attention because all hyponatremia patients present with hyponatremia on admission rather than developed it during the course of clinical management [6]. In addition, no existing evidence across a variety of disciplines and diseases demonstrated that correcting hyponatremia could result in better clinical end points so far [41].

Our study firstly demonstrated the association between hyponatremia and prognosis of RN, broadening the area of factors impacting on RN outcome and thus offered a new target on improving the prognosis in clinical practice. Nevertheless, this study still had some limitations. First, retrospective design left the unanswered question why hyponatremia patients presented increased progression of RN. Second, we did not collect the unavailable information regarding the irradiation strategy (radiation fields together with radiation dosages and schedule), though irradiation strategy has been demonstrated to be associated with occurrence rate of RN [42], which may also have impacts on the outcome of the patients. Third, we could not exclude other causes besides radiation necrosis that might also lead to hyponatremia in RN patients. For example, Table 1 shows a $2 \times$ higher frequency of cerebral infarction and a $4 \times$ higher frequency of pulmonary infarction associated with hyponatremia. Probably, the limited number of patients studied explains that these observations are not significant. In addition, results may have been biased by excluded patients with uncompleted data. Moreover, the data in the paper failed to answer that when and how often to use hyponatremia detection, and once hyponatremia is detected when the next MRI should be done. Additionally, our data showed that the median timing of the lowest serum sodium concentration was one month after the first MRI performed. The potential explanation maybe that one month after the first MRI performed, patients suffered from bad symptoms (which might be a sign of RN progression) and had to go to hospital to have medical check including blood examination which showed low concentration of sodium including hyponatremia, furtherly surpporting that hyponatremia is a potential predictor for the progression of patients with RN. However, more studies are warranted to confirm and explain this. Lastly, MR spectroscopy is a potential better choice to distinguish recurrent tumor and radiation injury in patients previously radiated for brain neoplasm [43]. However, since the MR spectroscopy data of the enrolled patients wasn't available, we had to choose conventional MR imaging.

\section{Conclusions}

In summary, hyponatremia is a common phenomenon in the hospitalized RN patients and acts as a potential predictor of progression, which has clinical significance that doctors need to pay more attention to the management of hyponatremia in RN patients. Large and prospective studies are needed to verify these findings and provide further evidence.

\section{Abbreviations}

ADH: Antidiuretic hormone; Cl: Confidence interval; CWS: Cerebral salt wasting syndrome; M: Median; MRI: Magnetic resonance imaging; OR: Odds ratio; RN: Radiation-induced brain necrosis; SD: Standard deviation; SIADH: The syndrome of inappropriate antidiuretic hormone secretion; VIF: Variance inflation factors

\section{Funding}

This study is supported by National Natural Science Foundation of China (No. 81572481, 81272197) to Ying Peng, International Collaboration Program of Universities in Guangdong Province (No.2012gjhz001) and the Key Project of Product, Study and Research of Guangzhou city (No. 201508020058) to Ying Peng, Grant KLB09001 from the Key Laboratory of Malignant Tumor Gene Regulation and Target Therapy of Guangdong Higher Education Institutes, Sun Yat-Sen University and Grant [2013]163 from Key Laboratory of Malignant Tumor Molecular Mechanism and Translational Medicine of Guangzhou Bureau of Science and Information Technology, National Natural Science Foundation of China (No. 81502167) and Natural Science Foundation of Guangdong Province (No. 2015A030313030) to Xiaoming Rong. 


\section{Availability of data and materials}

The data that support the findings of this study are available from Sun yat-sen Memorial Hospital but restrictions apply to the availability of these data, which were used under license for the current study, and so are not publicly available. Data are however available from the authors upon reasonable request and with permission of Sun yat-sen Memorial Hospital.

\section{Authors' contributions}

$\mathrm{HL}$ designed the study and wrote the manuscript. ZZ performed the analysis and interpretation of data as well as manuscript writing. XR and HW was responsible for the acquisition and interpretation of data and the manuscript revision. YP designed the study with $\mathrm{HL}$ and made a critical revision for the manuscript. All authors read and approved the final manuscript.

\section{Ethics approval and consent to participate}

The study was approved by the Institutional Review Board of Sun yat-sen Memorial Hospital. Patient's consents were waived by the Institutional Review Board of Sun yat-sen Memorial Hospital due to the retrospective design of the study.

\section{Consent for publication}

Not applicable.

\section{Competing interests}

The authors declare that they have no competing interests.

\section{Publisher's Note}

Springer Nature remains neutral with regard to jurisdictional claims in published maps and institutional affiliations.

\section{Author details}

'Department of Neurology, Sun Yat-sen Memorial Hospital, Sun Yat-Sen University, No. 107 West Yanjiang Road, Guangzhou 510120, China. ${ }^{2}$ State Key Laboratory of Ophthalmology, Zhongshan Ophthalmic Center, Sun Yat-sen University, Guangzhou, China. ${ }^{3}$ Guangdong Provincial Key Laboratory of Malignant Tumor Epigenetics and Gene Regulation, Sun Yat-sen Memorial Hospital, Sun Yat-sen University, Guangzhou, China.

Received: 30 October 2017 Accepted: 22 August 2018 Published online: 29 August 2018

\section{References}

1. Ho JH. An epidemiologic and clinical study of nasopharyngeal carcinoma. Int J Radiat Oncol Biol Phys. 1978;4(3-4):182-98.

2. Cole AM, Scherwath A, Ernst G, Lanfermann H, Bremer M, Steinmann D. Self-reported cognitive outcomes in patients with brain metastases before and after radiation therapy. Int J Radiat Oncol Biol Phys. 2013:87(4):705-12.

3. Greene-Schloesser D, Robbins ME, Peiffer AM, Shaw EG, Wheeler KT, Chan MD. Radiation-induced brain injury: a review. Front Oncol. 2012;2:73.

4. Castillo JJ, Glezerman IG, Boklage SH, et al. The occurrence of hyponatremia and its importance as a prognostic factor in a cross-section of cancer patients. BMC Cancer. 2016;16:564.

5. Hamaguchi S, Kinugawa S, Tsuchihashi-Makaya M, et al. Hyponatremia is an independent predictor of adverse clinical outcomes in hospitalized patients due to worsening heart failure. J Cardiol. 2014;63(3):182-8.

6. Kuramatsu JB, Bobinger $T$, Volbers $B$, et al. Hyponatremia is an independent predictor of in-hospital mortality in spontaneous intracerebral hemorrhage. Stroke. 2014:45(5):1285-91.

7. Bales J, Cho S, Tran TK, et al. The effect of hyponatremia and sodium variability on outcomes in adults with aneurysmal subarachnoid hemorrhage. World Neurosurg. 2016;96:340-9

8. Mapa B, Taylor BE, Appelboom G, Bruce EM, Claassen J, Connolly ES. Impact of hyponatremia on morbidity, mortality, and complications after aneurysmal subarachnoid hemorrhage: a systematic review. World Neurosurg. 2016;85: 305-14.

9. Brouwer MC, van de Beek D, Heckenberg SG, Spanjaard L, de Gans J. Hyponatraemia in adults with community-acquired bacterial meningitis. QJM. 2007;100(1):37-40.

10. Misra UK, Kalita J, Bhoi SK, Singh RK. A study of hyponatremia in tuberculous meningitis. J Neurol Sci. 2016;367:152-7.
11. Astaf'eva LI, Kutin MA, Mazerkina NA, et al. The rate of hyponatremia in neurosurgical patients (comparison between the data from the Burdenko Neurosurgical Instutite and the literature) and recommendations for the diagnosis and treatment. Zh Vopr Neirokhir Im N N Burdenko. 2016;80(1): $57-70$.

12. Moro N, Katayama Y, Igarashi T, Mori T, Kawamata T, Kojima J. Hyponatremia in patients with traumatic brain injury: incidence, mechanism, and response to sodium supplementation or retention therapy with hydrocortisone. Surg Neurol. 2007:68(4):387-93.

13. Hasan D, Wijdicks EF, Vermeulen M. Hyponatremia is associated with cerebral ischemia in patients with aneurysmal subarachnoid hemorrhage. Ann Neurol. 1990;27(1):106-8.

14. Lehmann L, Bendel S, Uehlinger DE, et al. Randomized, double-blind trial of the effect of fluid composition on electrolyte, acid-base, and fluid homeostasis in patients early after subarachnoid hemorrhage. Neurocrit Care. 2013;18(1):5-12.

15. Schrier RW, Sharma S, Shchekochikhin D. Hyponatraemia: more than just a marker of disease severity. Nat Rev Nephrol. 2013:9(1):37-50.

16. Hoorn EJ, Zietse R. Hyponatremia and mortality: moving beyond associations. Am J Kidney Dis. 2013;62(1):139-49.

17. Rahman M, Friedman WA. Hyponatremia in neurosurgical patients: clinical guidelines development. Neurosurgery. 2009;65(5):925-35. discussion 935-6

18. Diringer M, Ladenson PW, Borel C, Hart GK, Kirsch JR, Hanley DF. Sodium and water regulation in a patient with cerebral salt wasting. Arch Neurol. 1989;46(8):928-30

19. Spasovski G, Vanholder R, Allolio B, et al. Clinical practice guideline on diagnosis and treatment of hyponatraemia. Eur J Endocrinol. 2014;170(3): G1-47.

20. Tang $Y$, Rong $X$, Hu W, et al. Effect of edaravone on radiation-induced brain necrosis in patients with nasopharyngeal carcinoma after radiotherapy: a randomized controlled trial. J Neuro-Oncol. 2014:120(2):441-7.

21. Shah $R$, Vattoth $S$, Jacob $R$, et al. Radiation necrosis in the brain: imaging features and differentiation from tumor recurrence. Radiographics. 2012;32(5):1343-59.

22. Upadhyay A, Jaber BL, Madias NE. Incidence and prevalence of hyponatremia. Am J Med. 2006;119(7 Suppl 1):S30-5.

23. Kovesdy CP, Lott EH, Lu JL, et al. Hyponatremia, hypernatremia, and mortality in patients with chronic kidney disease with and without congestive heart failure. Circulation. 2012;125(5):677-84.

24. Cárdenas A, Solà E, Rodríguez E, et al. Hyponatremia influences the outcome of patients with acute-on-chronic liver failure: an analysis of the CANONIC study. Crit Care. 2014;18(6):700.

25. Palmer BF. Hyponatremia in patients with central nervous system disease: SIADH versus CSW. Trends Endocrinol Metab. 2003;14(4):182-7.

26. Isotani E, Suzuki R, Tomita K, et al. Alterations in plasma concentrations of natriuretic peptides and antidiuretic hormone after subarachnoid hemorrhage. Stroke. 1994;25(11):2198-203.

27. Tomida M, Muraki M, Uemura K, Yamasaki K. Plasma concentrations of brain natriuretic peptide in patients with subarachnoid hemorrhage. Stroke. 1998;29(8):1584-7.

28. von BP, Ankermann T, Eggert P, Claviez A, Fritsch MJ, Krause MF. Diagnosis and management of cerebral salt wasting (CSW) in children: the role of atrial natriuretic peptide (ANP) and brain natriuretic peptide (BNP). Childs Nerv Syst. 2006;22(10):1275-81

29. Fukui K, Inamura T, Nakamizo A, et al. Relationship between cardiac natriuretic peptide (ANP/BNP) and fluid intake in patients with subarachnoid hemorrhage. No To Shinkei. 2000;52(11):1019-23.

30. Yamada K, Goto A, Nagoshi H, Hui C, Omata M. Role of brain ouabainlike compound in central nervous system-mediated natriuresis in rats. Hypertension. 1994;23(6 Pt 2):1027-31.

31. Wijdicks EF, Vermeulen M, Hijdra A, van Gijn J. Hyponatremia and cerebral infarction in patients with ruptured intracranial aneurysms: is fluid restriction harmful. Ann Neurol. 1985;17(2):137-40.

32. Nardone R, Brigo F, Trinka E. Acute symptomatic seizures caused by electrolyte disturbances. J Clin Neurol. 2016;12(1):21-33.

33. Inamdar P, Masavkar S, Shanbag P. Hyponatremia in children with tuberculous meningitis: a hospital-based cohort study. J Pediatr Neurosci. 2016;11(3):182-7.

34. Lin YT, Huang CC, Chyau CC, Chen KC, Peng RY. Sixteen years post radiotherapy of nasopharyngeal carcinoma elicited multi-dysfunction along PTX and chronic kidney disease with microcytic anemia. BMC Urol. 2014;14:19.

35. Appelman-Dijkstra NM, Kokshoorn NE, Dekkers OM, et al. Pituitary dysfunction in adult patients after cranial radiotherapy: systematic review and meta-analysis. J Clin Endocrinol Metab. 2011;96(8):2330-40. 
36. Swart RM, Hoorn EJ, Betjes MG, Zietse R. Hyponatremia and inflammation: the emerging role of interleukin-6 in osmoregulation. Nephron Physiol. 2011;118(2):45-51.

37. Landgraf R, Neumann I, Holsboer F, Pittman QJ. Interleukin-1 beta stimulates both central and peripheral release of vasopressin and oxytocin in the rat. Eur J Neurosci. 1995;7(4):592-8.

38. Ohta M, Ito S. Hyponatremia and inflammation. Rinsho Byori. 1999;47(5):408-16.

39. Schnegg $\mathrm{Cl}$, Kooshki M, Hsu FC, Sui G, Robbins ME. PPARס prevents radiation-induced proinflammatory responses in microglia via transrepression of NF-KB and inhibition of the PKCa/MEK1/2/ERK1/2/AP-1 pathway. Free Radic Biol Med. 2012;52(9):1734-43.

40. Xue J, Dong JH, Huang GD, Qu XF, Wu G, Dong XR. NF-kB signaling modulates radiation-induced microglial activation. Oncol Rep. 2014;31(6):2555-60.

41. Konstam MA, Gheorghiade M, Burnett JC, et al. Effects of oral tolvaptan in patients hospitalized for worsening heart failure: the EVEREST outcome trial. JAMA. 2007;297(12):1319-31.

42. Jen YM, Hsu WL, Chen CY, et al. Different risks of symptomatic brain necrosis in NPC patients treated with different altered fractionated radiotherapy techniques. Int J Radiat Oncol Biol Phys. 2001;51(2):344-8.

43. Sundgren PC. MR spectroscopy in radiation injury. AJNR Am J Neuroradiol. 2009;30(8):1469-76.

Ready to submit your research? Choose BMC and benefit from:

- fast, convenient online submission

- thorough peer review by experienced researchers in your field

- rapid publication on acceptance

- support for research data, including large and complex data types

- gold Open Access which fosters wider collaboration and increased citations

- maximum visibility for your research: over $100 \mathrm{M}$ website views per year

At $\mathrm{BMC}$, research is always in progress.

Learn more biomedcentral.com/submissions 